Service social

\title{
Le Forum de la Conférence mondiale des femmes, Nairobi, Kenya, 10-19 juillet 1985
}

\section{Gisèle Legault}

Volume 34, numéro 2-3, 1985

L'organisation communautaire

URI : https://id.erudit.org/iderudit/706280ar

DOI : https://doi.org/10.7202/706280ar

Aller au sommaire du numéro

Éditeur(s)

École de service social de l'Université Laval

ISSN

1708-1734 (numérique)

Découvrir la revue

Citer ce document

Legault, G. (1985). Le Forum de la Conférence mondiale des femmes, Nairobi, Kenya, 10-19 juillet 1985. Service social, 34(2-3), 389-398.

https://doi.org/10.7202/706280ar d'utilisation que vous pouvez consulter en ligne.

https://apropos.erudit.org/fr/usagers/politique-dutilisation/ 


\section{COMMENTAIRES ET DOCUMENTS}

\section{Le Forum de la Conférence mondiale des femmes, Nairobi, Kenya, 10-19 juillet 1985}

\section{Gisèle Legault}

Ayant assisté et participé au Forum des Organismes non gouvernementaux de la Conférence mondiale des femmes, à Nairobi, je voudrais tenter ici d'en souligner quelques grandes lignes pour le bénéfice des travailleurs et travailleuses du champ des services sociaux et de la santé, les clientèles y étant majoritairement des femmes.

\section{Présentation générale}

\section{Contexte}

Le Forum des Organismes non gouvernementaux se tenait parallèlement à la Conférence des délégués gouvernementaux de pays convoqués par les Nations-Unies pour faire le bilan de la décennie des femmes : 1975-1985.

En 1975, à Mexico avait lieu la première Conférence mondiale des femmes où, à l'appel des Nations-Unies, plusieurs pays étaient conviés 
à soumettre des programmes susceptibles de favoriser le développement et l'égalité des femmes de ces sociétés. En 1980, à Copenhague, la deuxième Conférence avait pour but d'évaluer ces divers programmes, de faire le point et de proposer des orientations pour la seconde partie de la décennie. Mon propos n'est pas ici de faire état de la Conférence des délégués gouvernementaux puisque je n'y ai pas assisté et que d'autres documents en témoigneront, mais d'essayer de présenter certaines grandes lignes à partir de ma perception et de ma participation à ce Forum.

On y attendait 6000 femmes, il en est venu environ 12000 , de tous les pays du monde ; la ville de Nairobi et le campus de l'université en étaient submergés! Pendant sept jours, se sont déroulés simultanément une cinquantaine d'ateliers, et ce à raison de quatre par jour, soit un total d'environ mille pour toute la durée du Forum. J'estime avoir moi-même participé à des ateliers portant sur des sujets importants en même temps que diversifiés pour les femmes, ainsi : la production alimentaire, les mutilations sexuelles, la prostitution et le trafic des femmes, la situation des femmes dans des pays où l'oppression est manifeste ou prégnante tels: I'Amérique du Sud, l'Amérique centrale, la Palestine, l'Iran, l'Afrique du Sud.

\section{Perspective}

Si l'on considère la scène mondiale, on peut affirmer, à l'instar de Ruth Léger-Simard,' 'que les changements affectant les femmes depuis la deuxième guerre mondiale ont été extrêmement inégaux et au total modestes. Que ce soit en économie, en éducation, en santé, dans les pouvoirs publics, il n'y a pas de champ d'activités ni de pays où elles aient atteint une véritable égalité avec les hommes. Leurs luttes n'ont pas encore réussi à faire baisser leur niveau général de pauvreté. À travers le monde, les femmes sont encore sur-représentées parmi les pauvres, les analphabètes, les chômeuses, les sous-employées. Malgré la diversité d'expériences et de statut, elles se rencontrent toutefois de plus en plus sur un terrain commun, autour de cette trame de la vie des femmes qui traverse toutes les cultures. Elles partagent toutes ce sentiment d'inégalité d'opportunité, l'injustice de la "seconde place» imposée par la tradition, que ce soit dans la famille, ou sur les plans social, économique et politique.

Ce sentiment partagé a produit partout une volonté de changement; même s'il reste beaucoup à faire, surtout au niveau des attitudes, des coutumes, des lois, il y a eu beaucoup plus de fait depuis 
dix ans qu'au cours des décennies précédentes. Les rencontres mondiales, amorcées par les Nations-Unies, n'ont pas été étrangères à ces réalisations. Elles ont aidé les femmes à créer et cimenter des liens au-delà des barrières culturelles et géographiques, et le Forum de l'été 1985 à Nairobi a été particulièrement productif sur ce plan. Les sujets abordés sont vastes et souvent reliés à leur vie immédiate. Elles s'intéressent bien sûr à leurs droits civils mais, de plus en plus et avec un degré de sophistication et de pouvoir accru, aux structures sociales et aux priorités de la sphère publique qui affectent ces droits. À Nairobi, elles se sont intéressées, en priorité, aux questions de justice et d'égalité, de développement et de paix.

\section{Points saillants}

Je voudrais d'abord souligner l'atmosphère du Forum, qui en était une de disponibilité, d'ouverture aux idées, de partage d'expériences de femmes venant de pays et cultures très différents. Ces femmes se sont rencontrées, ont parlé directement et échangé sur tous les sujets, autrement que par gouvernements et médias officiels interposés. Régnait un climat de confiance et de coopération qui a permis ces échanges et engagé un processus de changement de situation qui se poursuivra bien au-delà de l'événement. À partir du principe mis de l'avant, à savoir le respect de l'expérience et des idées de l'autre, l'ouverture à sa réalité et le souci d'en arriver à des lignes communes d'action, les préoccupations et perspectives des femmes ont pu être abordées et discutées.

Il a aussi permis un contact direct avec les Africaines, relativement inconnues pour plusieurs d'entre nous. Les mythes qu'on peut entretenir à leur sujet ont pu être confrontés aux réalités décrites, et constatation a pu être faite qu'elles sont des femmes comme nous avec qui on peut discuter de tous les sujets nous touchant de façon commune, au même titre qu'on peut le faire en Amérique. L'exposé de leur situation nous a permis de toucher de près l'interdépendance de la situation des femmes au plan international. Entre autres, il nous a été permis de constater à maintes reprises que les positions prises par le Canada, sur le plan international, ont des conséquences précises sur les femmes du Tiers-Monde. On peut citer, comme exemple, le recours à la main-d'œuvre émigrée du Tiers-Monde lorsque les revendications des travailleuses d'ici deviennent trop exigeantes.

Lieu d'information exceptionnel pour les Africaines relativement peu au courant des réalités autres que les leurs, ce Forum, se déroulant en Afrique, a permis une participation imposante de celles-ci qui sont 
désormais des partenaires à part entière du Mouvement international des femmes.

Pour les Occidentales, le Forum a aussi permis une sensibilisation à la situation de celles qui vivent dans des pays où l'oppression est visible, manifeste et terrible. Il a créé une atmosphère où il était possible de les écouter, de les comprendre, d'appuyer leurs luttes et de concevoir des moyens de les soutenir une fois revenues chez nous.

Le degré de profondeur, de sérieux et de rigueur dans lequel les questions y ont été abordées est aussi à souligner; rien n'a été traité superficiellement et il s'en dégage une vision globale de certaines réalités sociétales. II n'y a pas de sujet qui ne concerne pas les femmes habituées à envisager les différents domaines de la vie de façon non compartimentée. Cette vision unificatrice du féminisme est probablement l'un des moyens les plus importants de l'édification de la paix dans le monde, l'un des thèmes les plus importants abordés lors de ce Forum.

\section{Quelques exemples d'ateliers}

\section{Femmes et développement}

Plusieurs des ateliers ont porté sur la participation des femmes à l'élaboration et à la conduite des projets de développement car, en dépit du rôle qu'elles jouent dans l'économie du Tiers-Monde en termes de travail concret et de temps, elles ne sont pas encore vraiment considérées dans les stratégies de développement.

Pour prendre l'exemple du Kenya, les femmes y sont majoritairement travailleuses dans le monde rural. Elles sont souvent les principales responsables et ce en nombre croissant, de la production alimentaire nécessaire à la survie des familles, les hommes s'adonnant aux cultures d'exportation, ayant émigré vers les villes par manque de travail ou ayant abandonné leur famille pour diverses raisons. Elles sont donc chargées de la production, de la transformation et de la préparation d'environ $80 \%$ de la nourriture nécessaire aux familles. L'état de santé de la population est donc directement relié à leur travail et aux conditions d'exercice de ce travail.

Les femmes du Tiers-Monde présentes au Forum, et tout particulièrement les Africaines, ont donc plaidé en faveur de projets de développement qui tiennent compte de leurs besoins et réalités spécifiques, qui soient axés entre autres sur les tâches qu'elles assument 
déjà, et sur des moyens de les faciliter, ainsi : l'accès à la terre, au crédit, à l'équipement, à la formation, aux moyens concrets et tangibles tels que l'eau, le combustible, les énergies. Dans l'état actuel des choses, elles s'occupent seules de l'agriculture de subsistance, activité invisible et non reconnue. On ne s'aperçoit pas, entre autres, que leur travail permet aux hommes de s'adonner à la culture d'exportation. Elles ont donc demandé que la priorité, en matière de développement, soit accordée aux questions qui touchent les femmes et qu'on fasse en sorte qu'elles soient les bénéficiaires des projets.

\section{Femmes et mutilations sexuelles}

Quand on pense aux mutilations sexuelles, on pense habituellement à l'excision et à l'infibulation encore largement pratiquées en Afrique, bien que de façon inégale selon les pays et les régions. Les Africaines, pour leur part, lient cette question à d'autres, qu'elles trouvent tout aussi importantes et autour desquelles elles veulent mener des luttes, ainsi celles pour l'abolition de la polygamie, du mariage forcé, de la stérilisation forcée et pour l'interruption volontaire et gratuite de la grossesse.

Ce qui est ressorti des débats, c'est que les Africaines sont contre les pratiques de mutilations sexuelles et estiment que ces pratiques ont des conséquences néfastes, dont : les infections résultant de la façon dont ces opérations sont menées, les problèmes autour des menstruations et des relations sexuelles, les difficultés d'accouchement, sans oublier la stricte question de l'intégrité du corps, de la jouissance sexuelle et de toutes les dimensions psychologiques encore largement inconnues. Elles estiment toutefois que l'ordre des luttes à mener et l'établissement des priorités leur reviennent, de même que les façons de s'y prendre ; pour certains pays, par exemple, il peut être plus urgent de s'occuper d'abord des questions de développement, d'alphabétisation que de mutilations sexuelles. Leur message est clair : " essayez de comprendre notre situation, informez et sensibilisez les femmes de vos pays à nos situations et laissez-nous la conduite des luttes".

Le Forum a été l'occasion, pour les Africaines de différents pays, de se connaître, de s'informer mutuellement et de s'organiser concrètement autour de plusieurs aspects, ${ }^{2}$ dont ceux des deux ateliers dont j'ai parlé précédemment. Contrairement aux femmes des pays occidentaux, elles commencent en quelque sorte leur décennie. Elles sont " tout yeux, tout oreilles" et ne laissent rien passer. Il sera intéressant de participer dans cinq ans, à la rencontre internationale déjà projetée à 
Madras, en Inde, en 1990, et d'y prendre connaissance des effets d'une telle mobilisation.

\section{Femmes et prostitution}

Cet atelier réunissait cinq collectifs de prostituées venues du Canada, des États-Unis, d'Angleterre, d'Australie et de Trinidad et Tobago et qui travaillent dans le sens de bâtir un regroupement international.

Ces participantes ont d'abord commencé par souligner que cette question concerne toutes les femmes parce qu'on réfère à une situation de travail difficile à laquelle chacune peut être amenée à recourir pour des raisons de survie économique. Elle a donc sa place dans ce Forum et doit préoccuper les féministes, ont-elles expliqué car, ce qui est immoral, ce n'est pas la prostitution en elle-même mais leur pauvreté à elles et celle des femmes tout court. La prostituée-type, à travers le monde, est en effet une femme seule, majoritairement de race noire et avec charge d'enfants. Son travail est donc avant tout une réponse à sa pauvreté en attendant de trouver une meilleure alternative. Ces collectifs ont demandé le support du Forum pour une décriminalisation de la prostitution et l'abolition des lois les empêchant de travailler, ce qui éviterait la répression, sans toutefois en rendre son institution légitime. Elles ne sont cependant pas en faveur de la légalisation de la prostitution puisqu'elle profiterait davantage aux proxénètes qu'aux femmes.

Un parallèle est fait entre le travail ménager gratuit et la prostitution. Si le travail fait par les femmes n'est pas payé, il est pris pour acquis et non reconnu socialement. On peut disposer de leur force de travail et aussi de leur corps. La perspective de lutte des collectifs est donc semblable à celle de groupes italiens et anglais des années 19721975 luttant pour une meilleure rétribution du travail ménager : on exigeait une reconnaissance du travail accompli en le payant convenablement, et on revendiquait des conditions décentes d'hygiène et de santé, sans toutefois ancrer les femmes dans celui-ci ou énoncer que ce sont elles qui doivent le faire. En attendant que d'autres choix soient possibles, on soutient cette perspective de reconnaissance concrète et tangible du travail fait.

Relié au sujet de la prostitution, celui de l'esclavage sexuel ou du trafic des femmes à travers le monde qui a fait l'objet d'un atelier d'une demi-journée. Les travaux sur cette question ont commencé à la conférence internationale de la mi-décennie, à Copenhague, en 1980, pour se poursuivre plus intensivement lors d'une rencontre mondiale à 
Rotterdam, aux Pays-Bas, en $1983 .{ }^{3}$ À la suite de cette rencontre, un Réseau féministe international contre l'esclavage sexuel a été mis sur pied et son bilan a fait l'objet de l'atelier à Nairobi, réunissant des représentantes d'Afrique, de Thaïlande, des Philippines, de l'Inde et de la République dominicaine. A été défini comme esclavage sexuel toute situation où une femme qui ne peut s'en évader est psychologiquement malmenée et sexuellement exploitée. Ainsi, l'esclavage sexuel s'applique aux femmes victimes de "tourisme sexuel" et du "marché du mariage" tel qu'il se pratique actuellement entre des agences privées d'Allemagne et du Sud-Est asiatique, aussi bien qu'à la prostitution forcée sur les bases militaires.

La vision féministe de l'esclavage sexuel ne s'attaque pas aux prostituées elles-mêmes mais à la prostitution en tant qu'institution, envisagée comme le résultat du pouvoir patriarcal, de la violence sexuelle et de l'asservissement. En conséquence, on fait "des rapports sexuels un droit masculin, qu'ils soient achetés, vendus, contraints par le viol ou la violence ou plus subtilement obtenus par le harcèlement $"{ }^{4}$ Cela signifie qu'on admet que la sexualité et le corps des femmes peuvent être transformés en marchandise que l'on vend.

Les panellistes de l'atelier ont parlé de la situation des femmes et de l'exploitation de la prostitution dans leur région. Une salle comble avec traduction en quatre langues écoutait attentivement à mesure qu'un sentiment souvent expérimenté dans de nombreuses réunions féministes commençait à se manifester : le sentiment d'un but commun rompant l'isolement qui entoure bien des luttes féministes ou apportant une nouvelle prise de conscience et une énergie renouvelée. Ainsi, à titre d'exemple, des féministes de Thaïlande et des Philippines ont décrit les agences privées d'Allemagne qui organisent des mariages forcés avec des "épouses choisies sur catalogue" provenant de leur pays, mariages qui sont la porte d'entrée à des réseaux de prostitution. Elles ont aussi souligné les mythes qui ont cours quant à la docilité et la passivité des Asiatiques en une période où les Occidentales seraient devenues trop "libérées". Une féministe indienne a dénoncé le devadasis dans son pays, système qui consiste pour les familles à consacrer chaque année 5000 fillettes de huit à dix ans à un temple; les familles reçoivent une rémunération de la part d'un propriétaire de bordel et les fillettes sont amenées à Bombay ou dans la région du Golfe pour la prostitution. Sous couvert de rites anciens, les raisons actuelles de cette consécration de toute jeunes filles se résument à un recrutement pour la prostitution.

Les travaux de cet atelier se continueront à travers le Réseau féministe international contre l'esclavage sexuel et la traite des femmes, 
et luttera principalement contre la violence et leur exploitation sexuelle. Les premières actions seront dirigées contre la prostitution, la traite des femmes, le tourisme sexuel et leurs rapports avec la violence tels le viol, les mutilations sexuelles, l'inceste, les sévices, les mariages imposés, la dot et l'achat de l'épouse, la pornographie et la torture des prisonnières politiques.

\section{Femmes palestiniennes, femmes d'Afrique du Sud}

Les médias ont beaucoup fait état des débats qui ont eu lieu sur la condition des femmes dans un contexte politique conflictuel, par exemple les Palestiniennes. II est vrai que, dans certains ateliers, les échanges ont été tendus et qu'il a été impossible de poursuivre les travaux. J'ai toutefois assisté moi-même à deux ateliers où les principes du Forum ont été respectés, à savoir où les idées et l'expérience de chaque femme ont été respectées et où un climat d'ouverture accueillait ces mêmes idées, le Forum n'étant pas un lieu d'affrontement mais d'échange et d'écoute. J'ai constaté là qu'Israéliennes et Palestiniennes pouvaient s'écouter, exposer leurs points de vue respectifs sur la situation des unes et des autres et tenter d'en arriver à une entente. Bien sûr, les discussions n'ont pas été faciles et des opinions et sentiments souvent très forts ont bloqué la communication à maintes reprises, les positions très partisanes de femmes d'autres pays arabes ou des Iraniennes ont aussi contribué à complexifier les débats. Il en reste tout de même que des échanges positifs ont pu avoir lieu, à Nairobi, et qu'un progrès certain a pu être noté en comparaison avec Copenhague.

La situation en Afrique du Sud a bien sûr fait l'objet de plusieurs débats, d'autant plus que nous étions en Afrique. Aucune représentante du gouvernement de Prétoria n'était à la Conférence, non plus que des femmes d'organismes non gouvernementaux sud-africains. De nombreuses militantes de I'A.N.C. (Congrès national africain, principal mouvement anti-apartheid) et de la S.W.A.P.O. (Organisation de libération du peuple du Sud-Ouest-Africain vivant en dehors de l'Afrique du Sud), sont toutefois venues nous exposer la situation des femmes vivant sous l'apartheid. Dans ce pays du Tiers-Monde où le minimum de justice sociale n'existe pas pour la majorité noire de la population, leur condition se trouve principalement affectée par la domination et le pouvoir qu'exerce le régime en place sur cette population, mais aussi par les conditions de vie et de travail spécifiques des femmes, entre autres par l'impossibilité de profiter de l'accès à la terre, à l'équipement et aux ressources dont il a été fait mention auparavant. 
Des ateliers ont présenté la situation d'autres femmes qui vivent écrasées par une domination et un pouvoir qui se manifeste de façon crue et grossière, entre autres en Argentine, au Chili de Pinochet, au El Salvador et au Guatémala, pour ne mentionner que les ateliers auxquels j'ai personnellement assisté.

\section{Conclusion}

Le Forum de Nairobi a été une formidable occasion de rencontres de femmes du monde entier autour de problèmes concrets reliés à la technologie, la santé, l'éducation, l'agriculture, le développement, l'organisation et, à ce titre, il a été un succès. De toutes cultures, de toutes nationalités, elles ont pu s'informer de leurs réalités respectives, se sensibiliser à l'impact de ces réalités, trouver des façons concrètes d'appuyer les luttes menées par les unes et les autres et continuer de les soutenir une fois revenues dans leur pays.

En résumé, Nairobi a permis d'affirmer le caractère irréversible et international du Mouvement des femmes. Toutes se sont rencontrées autour d'une volonté commune de modifier leur situation, leur féminisme se définissant de façon large en une vision par les femmes de leur avenir et du développement de leurs collectivités respectives. La vitalité de ce mouvement se traduira au cours des prochains mois en une multitude de regroupements spécifiques reliés à des tâches concrètes autour de plusieurs thèmes dont ceux déjà mentionnés ici et dans d'autres articles. Un groupe québécois sur la thématique Femmes et Développement sera formé afin de structurer une participation plus active lors d'une prochaine rencontre internationale, notre présence, ainsi que celle des Canadiennes, ayant été plutôt discrète cette fois-ci. Des objectifs ont été fixés pour les cinq prochaines années, souvent même jusqu'à l'an 2000, avec l'idée de tout réévaluer en 1990, en Inde (rencontre non encore confirmée).

Je n'oublierai pas cette belle finale sur le campus de l'Université de Nairobi le vendredi 19 juillet, où plusieurs groupes de femmes africaines nous ont donné le spectacle de leurs productions culturelles variées et magnifiques. Je me rappelle spécialement l'apothéose qu'a été ce spectacle musical d'une Américaine noire qui improvisait sur ce qu'avait été pour elle ce Forum, sur l'identité noire en Amérique comme en Afrique, et son identité retrouvée dans un continent où l'on est fière d'être noire. Son poème, en finale, "Black is... the color of my skin", récité de façon superbe, forte, fière et glorieuse, clôturait bien cet événement historique. 


\section{Notes et références}

1 Ruth LÉGer-Simard, Women: A World Survey, Washington, World Priorities, 1985.

2 Les pôles d'organisation ont été ceux des femmes des pays francophones dont: la Côte d'Ivoire, le Cameroun, le Mali, le Sénégal, le Burkina Faso et celui des femmes des pays anglophones d'Afrique dont : le Kenya, I'Éthiopie, la Tanzanie, le Nigéria, et j'en oublie.

3 Voir, à ce sujet, un numéro spécial de Nouvelles questions féministes, "Féminisme international : réseau contre l'esclavage sexuel ", no 8, 1984.

4 Id. 